patients and other in 13 patients. Seven patients started HPN prior to 2000. Between 2000 and 2011, 61 patients started HPN and 35 patients stopped. Forty-three patients started HPN following gastrointestinal surgery and in 20 of these, HPN was needed because of a surgical complication (nine small bowel fistulae; 10 intraabdominal sepsis leading to high output stoma; one bowel obstruction). Thirty-five patients stopped HPN. Seventeen patients died - nine of the underlying disease, four of unrelated cause, two of HPN complications and two of both HPN complications and the underlying disease. Seventeen patients stopped HPN after reconstructive surgery, HPN was stopped due to complications/lack of progress in one patient and one patient stopped HPN because of more effective medical treatment. Three other patients reduced their dependence on HPN after surgery (fewer nights per week or lower volume). Of the twenty patients who had HPN because of surgical complication, ten patients stopped HPN because of further surgery, five patients have ongoing HPN with no intention for further surgery, two patients have died (one of the underlying malignancy and the other of unrelated cause) and three patients are likely to have reconstructive surgery in the future. None of the patients who came to reconstructive surgery after a period of HPN died after their surgery.

Conclusion Although the complications of surgery may lead to home parenteral nutrition, surgery may also be useful in reconstruction of the GI tract and this may lead to stopping HPN. It is important that surgeons are involved in the care of HPN patients, as they may be more conscious of the possibility of reconstruction.

Competing interests None declared.

\section{OC-073 CALCULATING THE COST-EFFECTIVENESS OF A NUTRITION SUPPORT TEAM AT A BUSY DISTRICT GENERAL HOSPITAL}

doi:10.1136/gutjnl-2012-302514a.73

M Arsanious, ${ }^{*}$ K Eaton, M Burke, 0 lyowu, S Gupta, A Tarn. Croydon University Hospital, London, UK

Introduction NICE Nutrition Support In Adults (2006) recommends that all Trusts should have a multidisciplinary Nutrition Support Team (NST). Austin \& Stroud (2007) suggest its implementation can improve parenteral nutrition (PN) services and potentially reduce $\mathrm{PN}$ usage by up to $25 \%$. At Croydon University Hospital (CUH), a multidisciplinary NST was set up in October 2010, consisting of a Consultant Chemical Pathologist, Consultant Gastroenterologist, dietians and pharmacists. It combined the previous $\mathrm{PN}$ team with the enteral nutrition (EN) team with the aim to optimise the EN of patients thereby reducing inappropriate PN usage as recommended by NCEPOD 2010. We aimed to investigate the use of PN 1 year prior to the implementation of a multidisciplinary NST and compare this to the use of PN 1 year post-implementation at $\mathrm{CUH}$.

Methods Case notes of patients who had received PN at CUH between 1st October 2009 and 30th September 2010 were obtained and a retrospective analysis of each patient's duration on PN was made and compared to prospective data, obtained from the start of the introduction of a multidisciplinary NST on 1st October 2010, until September 30th 2011. The final cost of PN was obtained from the hospital pharmacy and cost-savings were calculated.

Results Data obtained before and after implementation of NST: The total cost of PN per day is $£ 156.60$. The median number of days was reduced by 1 day. In the year following the introduction of the NST, 60 patients received PN (saving 60 days of PN), 12 patients were successfully prevented from receiving $\mathrm{PN}$ (saving 72 days of $\mathrm{PN}$ ). The total number of $\mathrm{PN}$ days saved was 132, resulting in a total cost saving of $£ 20671.20$. This does not take into consideration the range 16-78 years) had HPN supervised by our hospital during 11 years. The underlying disease was Crohn's disease in 26 patients, mesenteric ischaemia in 12 patients, malignancy in seven patients, motility disorders in eight patients, radiation enteritis in seven 